Lymphome

Z Rheumatol 2017 · 76 (Suppl 2):S46-S56 https://doi.org/10.1007/s00393-017-0383-4

๑) Springer Medizin Verlag GmbH 2017

\section{Redaktion}

H.-P. Tony, Würzburg

CrossMark

\author{
A. Rubbert-Roth' $\cdot$ J. T. Bittenbring ${ }^{2} \cdot$ G. Assmann ${ }^{2}$ \\ ${ }^{1}$ Medizinische Klinik I der Universität Köln, Köln, Deutschland \\ ${ }^{2}$ Medizinische Klinik I für Onkologie, Hämatologie, Klinische Immunologie und Rheumatologie, \\ Universitätsklinikum des Saarlandes, Homburg/Saar, Deutschland
}

\title{
Lymphome bei rheumatischen Erkrankungen
}

b) Lymphomrisiko unter Biologika
und „small molecules“. 2 Abschnitte. Im ersten werden die 4 bedeutsamsten Entitäten aus dem Formenkreis der Lymphome inklusive der chronisch lymphatischen Leukämie dargestellt:

- Hodgkin-Lymphom,

- Non-Hodgkin-Lymphome (NHL):

- diffus großzelliges B-Non-Hodgkin-Lymphom (DLBCL),

- chronisch lymphatische Leukämie,

- follikuläres Lymphom.

Dabei liegt der Schwerpunkt in der Darstellung der modernen Therapiekonzepte, die sich streng an den histologischen Subtypen und am Krankheitsstadium inklusive spezifischer Prognosefaktoren ausrichten. In dieser Weise wurde in den letzten 10 Jahren eine erhebliche Steigerung der Krankheitskontrolle sowie der Heilungsraten verschiedener Lymphomerkrankungen erreicht.

Der zweite Abschnitt behandelt die Assoziation von Lymphomen mit rheumatischen Erkrankungen und ihrer antirheumatischen Therapie. Im Einzelnen sind die Themen wie folgt gegliedert:

- Lymphome bei entzündlich rheumatischen Erkrankungen:

a) Lymphome und rheumatoide Arthritis,

b)Lymphome und Sjögren-Syndrom, c) Lymphome bei anderen Kollagenosen,

d)Lymphome bei Vaskulitiden;

- Lymphomrisiko unter DMARDs („disease modifying antirheumatic drugs"):

a) Lymphomrisiko unter konventionellen DMARDs,
Die Thematik wird dabei vorwiegend aus umfangreichen Registerdaten und Studiendaten zur medikamentösen Therapie mit Antirheumatika entwickelt. Der Beitrag soll unter anderem die aktuellen Erkenntnisse aufzeigen, wie das Risiko einer Lymphomentwicklung durch die rheumatische Krankheitsaktivität und durch ihre spezifische antirheumatische Therapie einzuschätzen ist.

\section{Lymphomentitäten}

\section{Hodgkin-Lymphom}

Das Hodgkin-Lymphom tritt im frühen Erwachsenenalter auf, allerdings gibt es auch im hohen Lebensalter einen zweiten Inzidenzgipfel. Die Gesamtinzidenz ist 2-3/100.000 Einwohner [13].

Typischerweise treten Lymphknotenschwellungen am Hals, axillär oder in der Leiste auf, oft sind auch nur mediastinal, selten mesenteriale Lymphknoten betroffen. Begleitend können B-Symptome auftreten (Nachtschweiß mit Wäschewechsel, Gewichtsverlust über $10 \%$ des Körpergewichts und Fieber $>38,3^{\circ} \mathrm{C}$ ). Selten treten Pruritus und alkoholinduzierte Schmerzen in den Lymphknoten auf.

\section{》) Typischerweise treten \\ Lymphknotenschwellungen am Hals, axillär oder in der Leiste auf}

Eine chirurgische Lymphknoten- oder Exzisionsbiopsie ist zur histologischen Beurteilung notwendig. Man unter- scheidet den häufigsten Subtyp noduläre Sklerose von dem selteneren Lymphozyten-prädominanten, Lymphozytenarmen und gemischtzelligen Subtyp.

Zur Ausbreitungsdiagnostik „Staging " wird international eine PETCT (PositronenemissionstomographieComputertomographie) empfohlen, in Deutschland muss hier jedoch eine Kostenübernahme zunächst beantragt werden. Das Stadium wird nach AnnArbor festgelegt ([8]; • Tab. 1).

Zusätzlich zum Stadium sind Risikofaktoren definiert: Blutsenkung $>50 \mathrm{~mm} / \mathrm{h}$ mit B-Symptomen, $30 \mathrm{~mm} / \mathrm{h}$ ohne B-Symptome, Mediastinaltumor $>1 / 3$ Thoraxdurchmesser und $>3$ Lymphknotenregionen und Extranodalbefall [13].

Die Standardtherapie im limitierten Stadium I/II ohne Risikofaktoren sind 2 Zyklen ABVD (Doxorubicin, Bleomycin, Vincristin, Dacarbazine), gefolgt von einer Strahlentherapie mit 20 Gy auf die betroffene Region („involved-field“). Bei Vorliegen von Risikofaktoren werden 4 Zyklen ABVD gegeben, gefolgt von einer $30 \mathrm{~Gy}$ Involved-field-Strahlentherapie. Bei jüngeren Patienten $<60$ Jahre haben sich in einer deutschen Studie 2 Zyklen ABVD + 2 intensivere Zyklen

\begin{tabular}{ll}
$\begin{array}{l}\text { Tab. } 1 \\
\text { tomen }\end{array}$ & Suffix B bei vorhandenen B-Symp- \\
\hline Stadium I & 1 Lymphknotenregion \\
\hline Stadium II & $\begin{array}{l}>1 \text { Lymphknotenregion auf } \\
\text { einer Seite des Zwerchfells }\end{array}$ \\
\hline Stadium III & $\begin{array}{l}>1 \text { Lymphknotenregion auf } \\
\text { beiden Seiten des Zwerchfells }\end{array}$ \\
\hline Stadium IV & $\begin{array}{l}\text { Diffuser Organbefall: Knochen- } \\
\text { mark, Peritoneum }\end{array}$ \\
\hline
\end{tabular}




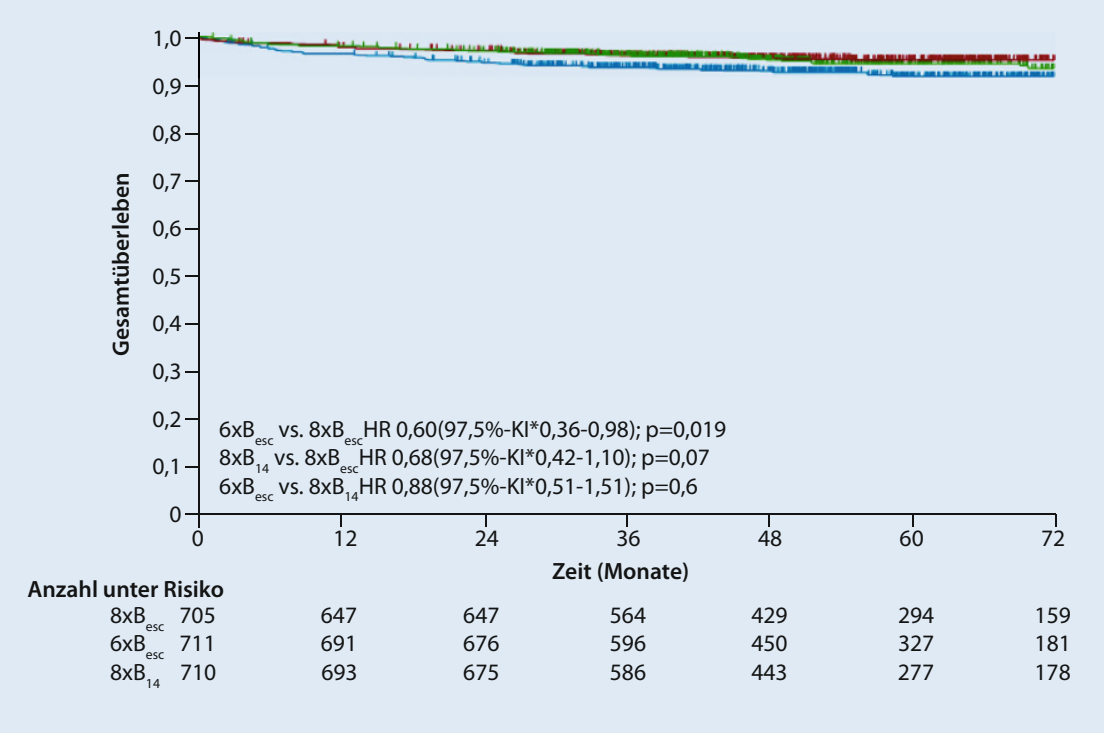

Abb. 1 A Kaplan-Meier-Kurve (unter verschiedenen BEACOPP-Protokollen). (Aus [15])

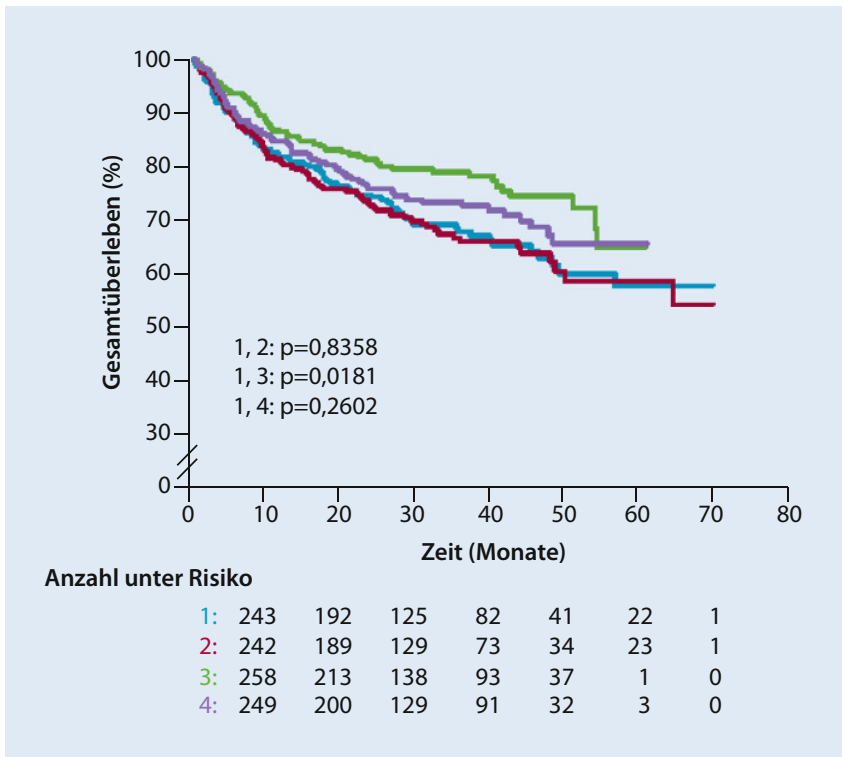

Abb. $2<$ KaplanMeier-Kurve: verschiedene CHOP-Protokolle mit und ohne Rituximab. (Aus [44])

BEACOPP (Bleomycin, Etoposid, Doxorubicin, Cyclophosphamid, Vincristin, Prednison, Procarbazin) als wirksamer herausgestellt. In fortgeschrittenen Stadien werden 6 bis 8 Zyklen ABVD gegeben oder bei jüngeren Patienten $<60$ Jahre eine intensivere Chemotherapie nach dem BEACOPP-Schema. Nach Abschluss der Therapie werden Restbefunde $>1,5 \mathrm{~cm}$ bestrahlt oder nur PET-positive Restbefunde [15]. In dieser Indikation ist die PET zugelassen. Die Prognose ist exzellent, innerhalb von Studien ist das Gesamtüberleben um $90 \%$ (• Abb. 1). tablen Patienten oder als Erhaltungstherapie nach autologer Transplantation mit sehr hohem Rezidivrisiko: Die Risikofaktoren sind

- refraktäres Hodgkin-Lymphom,

- Rezidiv innerhalb von 12 Monaten,

- Extranodalbefall [40].

Das CD30-Antigen wird auch von anaplastisch großzelligen Lymphomen und anderen T-Zell-Lymphomen exprimiert, was auch hier das therapeutische Armamentarium erweitert.

Weitere Optionen bestehen mit den Immun-Checkpoint-Inhibitoren Nivolumab und Pembrolizumab, die für die Indikation des rezidivierten HodgkinLymphoms nach autologer Stammzelltransplantation und Brentuximab-Vortherapie oder bei nichttransplantablen Patienten nach Brentuximab-Vortherapie zugelassen wurden.

Der monoklonale Antikörper Rituximab, der gegen das CD20-Antigen auf allen B-Zellen gerichtet ist, kann bei Lymphozyten-prädominantem Hodgkin eingesetzt werden.

Aktuell untersucht die deutsche Studiengruppe für das Hodgkin-Lymphom (GHSG) in der HD-21-Studie, ob Brentuximab 2 Chemotherapeutika (Bleomycin und Procarbazin) ausgleichen kann.

\section{Non-Hodgkin-Lymphome}

Die große Vielfalt der Non-Hodgkin Lymphome (NHL) wird in der WHOKlassifikation der hämatologischen Neoplasien dargestellt, die Ende 2016 aktualisiert wurde. Für eine Kurzübersicht soll hier nur auf Prinzipien und häufige Entitäten eingegangen werden.

Non-Hodgkin-Lymphome werden in B-Zell- und T-Zell-Lymphome eingeteilt, wobei in den Lymphknoten häufiger die B-Zell-Lymphome und an der Haut häufiger T-Zell-Lymphome auftreten. Bei den B-Zell-Lymphomen werden aggressive, schnell wachsende von den langsam wachsenden, indolenten Lymphomen abgegrenzt.

\section{Diffus großzelliges Lymphom}

Das diffus großzellige Lymphom (DLB$\mathrm{CL}$ ) ist das häufigste aggressive NHL mit einer Inzidenz von 4/100.000 Einwoh- 
ner. Es entsteht öfter ab der 4. Lebensdekade, kann aber in jedem Alter auftreten. Oft wird eine rasch progrediente Lymphknotenschwellung bemerkt, aber die klinische Manifestation ist sehr mannigfaltig, und fast jede Raumforderung kann histologisch ein DLBCL sein. Oft sind CT-gesteuerte Stanzbiopsien ausreichend aussagekräftig, eine Zytologie aus Feinnadelaspiration ist nicht ausreichend. Wenn möglich, sollte eine Bestätigung der Histologie durch einen Referenzpathologen angestrebt werden, um die definitive Entität zu klären.

\section{》) Das diffus großzellige Lymphom ist das häufigste aggressive Non-Hodgkin- Lymphom}

Eine kontrastgestütztes CT oder PETCT sollte durchgeführt werden für Manifestationen im Mesenterium, und zur Beurteilung von Gefäßkompression z. B. im Mediastinum ist die kontrastmittelgestützte konventionelle CT immer noch überlegen [58].

Die Prognose wird durch den internationalen prognostischen Index (IPI) (- Tab. 2) bestimmt [51].

Die Standardchemotherapie ist R-CHOP (Rituximab, Cyclophosphamid, Doxorubicin, Vincristin, Prednison) alle 21 Tage für 6 Zyklen. Der monoklonale Antikörper Rituximab hat die Prognose deutlich verbessert, sodass heute selbst in prognostisch ungünstiger Situation über die Hälfte der Patienten überlebt (• Abb. 2), in prognostisch günstigen ist das Überleben sogar nahezu $100 \%$.

Zusätzlich zur Chemotherapie sollten Extranodalbefälle und Regionen mit Tumoren $>7,5 \mathrm{~cm}$ (,bulky“) bestrahlt werden. Vermutlich ist dies nicht mehr notwendig, wenn eine PET-CT keine metabolische Restaktivität zeigt. Die deutsche Studiengruppe für hochmaligne Lymphome (DSHNHL) konnte zeigen, dass eine dosisdichtere Applikation von CHOP alle 14 Tage das Gesamtüberleben verbessert, wobei eine längere Exposition von Rituximab über die Chemotherapie hinaus eine Verbes-

Z Rheumatol 2017 · 76 (Suppl 2):S46-S56 https://doi.org/10.1007/s00393-017-0383-4

(c) Springer Medizin Verlag GmbH 2017

\section{A. Rubbert-Roth · J. T. Bittenbring · G. Assmann \\ Lymphome bei rheumatischen Erkrankungen}

\section{Zusammenfassung}

Das Risiko für die Entwicklung eines Lymphoms ist bei verschiedenen entzündlichen Systemerkrankungen wie der rheumatoiden Arthritis, dem Sjögren-Syndrom oder dem systemischen Lupus erythematodes erhöht. Studien zu Patienten mit rheumatoider Arthritis und Sjögren-Syndrom zeigen, dass die Lymphominzidenz mit der Schwere bzw. Aktivität der Erkrankung assoziiert ist, andererseits handelt es sich gerade bei den Lymphomen um Erkrankungen, die bevorzugt bei immunsupprimierten Patienten auftreten. Daher ist die Kenntnis dieser Krankheitsbilder, ihrer Therapie und Prognose auch für den Rheumatologen wesentlich. Bezüglich der konventionellen Basistherapeutika und Biologika gibt es erfreulicherweise keine Hinweise, dass diese Therapien mit einem erhöhten Lymphomrisiko einhergehen. Die Daten zur Frage, welche antirheumatische Therapie beim Vorliegen eines Lymphoms in der Vorgeschichte zu favorisieren ist, sind limitiert, da Patienten mit Malignomen in der Vorgeschichte nicht in klinische Studien eingeschlossen werden und in Registern ein Bias bezüglich Therapieselektion berücksichtigt werden muss. Im Einzelfall muss daher die Entscheidung bezüglich des therapeutischen Vorgehens interdisziplinär - gemeinsam mit dem behandelnden Hämatologen - erfolgen.

\section{Schlüsselwörter}

Rheumatoide Arthritis · Sjögren-Syndrom . Systemischer Lupus erythematodes . Basistherapeutika · Biologika

\section{Lymphoma in rheumatic diseases}

\section{Abstract}

Various systemic inflammatory diseases, such as rheumatoid arthritis (RA), Sjögren's syndrome and systemic lupus erythematosus (SLE) are associated with an increased risk for the development of lymphomas. Studies on patients with RA and Sjögren's syndrome have shown that there is a clear association of the incidence of lymphoma with the severity and activity of the disease and lymphomas in particular are diseases which preferentially occur in immunosuppressed patients; therefore, knowledge of the different lymphoma subtypes, their prognosis and treatment options are important for rheumatologists. Currently, there is no evidence for an increased risk of lymphoma with the available conventional basis therapies or biologic disease-modifying antirheumatic drugs (DMARDs). The decision on how to treat a patient with previous lymphoma who requires antirheumatic treatment is more difficult as patients with previous malignancies are not included in clinical studies and in registries a bias with respect to patient selection must be taken into consideration. Decisions on the treatment approach, therefore need to be individualized and interdisciplinary management together with the treating hematologist is warranted.

\section{Keywords}

Rheumatoid arthritis - Sjogren's syndrome · Systemic lupus erythematosus - DMARDs . Biologics serung brachte. In der RICOVER-Studie waren 6-mal R-CHOP-14 + 8 Gaben Rituximab der beste Arm [44], eine Erhaltungstherapie über 2 Jahre bringt jedoch keinen Vorteil [31].

Im Rezidiv sind 3 Zyklen einer Chemotherapie nach dem DHAP (Dexamethason, Hochdosis-AraC, Cisplatin)Schema [20] oder GDP (Gemcitabin, Cisplatin, Dexamethason) [9], gefolgt von einer autologen Stammzelltransplantation Standard. Cisplatin kann wahrscheinlich durch Oxaliplatin er- setzt werden, aber eine große Phase-IIIStudie dazu fehlt.

Die besonders schlechte Prognose von frühzeitigen Rezidiven (1 Jahr) und refraktären Patienten kann zur Erwägung einer allogenen Stammzelltransplantation führen [21].

$\mathrm{Zu}$ Therapieverbesserung werden mehrere Ansätze verfolgt. Die Optimierung der Gaben von Rituximab wird zurzeit von der DSHNHL untersucht [45]. Durch Genexpressionsanalyse konnten 2 Subtypen des DLBCL identifiziert 
Tab. 2 Risikostratefizierung bei hochmalignen Lymphomen mit Berechnung des IPI (Internationaler prognostischer Index)

\begin{tabular}{|c|c|}
\hline Risikofaktor & Punkt für \\
\hline Alter & $>60$ Jahre \\
\hline $\begin{array}{l}\text { Allgemeinzustand nach } \\
\text { ECOG }\end{array}$ & $>2$ \\
\hline $\begin{array}{l}\text { Serumlaktatde- } \\
\text { hydrogenase }\end{array}$ & $\begin{array}{l}\text { >Normalwert } \\
\text { des Labors }\end{array}$ \\
\hline Stadium nach Ann Arbor & III/IV \\
\hline Extranodalbefälle & $\geq 2$ \\
\hline \multicolumn{2}{|c|}{$\begin{array}{l}\text { Ein IPI (internationaler prognostischer Index) } \\
>2 \text { gilt als Hochrisiko } \\
\text { ECOG Eastern Cooperative Oncology Group }\end{array}$} \\
\hline
\end{tabular}

Tab. 4 Die Prognose wird durch Risikofaktoren mit Bestimmung des CLL (chronisch lymphatische Leukämie)-IPI (internationaler prognostischer Index) abgebildet

\section{Risikofaktor}

TP53 mutiert

IGVH unmutiert

B2-Mikroglobulin $>3,5 \mathrm{mg} / \mathrm{l}$

Stadium Binet B/C

Alter $>65$

CLL-IPI-Gruppe

Niedriges Risiko

Intermediär

Hoch

Sehr hoch

IGVH Immunglobulin-VH-Gen
Tab. 3 Einteilung der chronisch lymphatischen Leukämie (CLL) nach Binet [4]

\begin{tabular}{|c|c|}
\hline Stadium & Kriterien \\
\hline \multirow[t]{2}{*}{ A } & $\mathrm{Hb} \geq 10 \mathrm{~g} / \mathrm{dl}$ \\
\hline & Thrombozyten $\geq 100 / n l$ \\
\hline \multirow[t]{2}{*}{ B } & $\begin{array}{l}\geq 3 \text { Lymphknotenregionen } \\
\text { vergrößert }\end{array}$ \\
\hline & Blutbild wie Binet $A$ \\
\hline \multirow[t]{2}{*}{ C } & $\mathrm{Hb}<10 \mathrm{~g} / \mathrm{dl}$ \\
\hline & Thrombozyten $<100 /$ nl \\
\hline
\end{tabular}

mittels Schnittbildverfahren bei Erstdiagnose zur Stadieneinteilung (• Tab. 3) erfolgen.

Die zyto- und molekulargenetische Analyse der CLL muss ergänzt werden, um prognostisch ungünstige Formen der CLL mit Deletion 17p bzw. TP53Mutation zu identifizieren. Zur besseren Prognoseabschätzung werden diese genetischen Information in den neuen IPI der CLL aufgenommen, den sog. CLLIPI ([30]; • Tab. 4).

Der Behandlungsbeginn richtet sich nach direkt CLL-bedingten Komplikationen wie nicht autoimmunbedingter $\mathrm{Zy}$ topenie oder symptomatischer Hepatosplenomegalie/Lymphadenopathie.

Aufgrund des durchschnittlich höheren Patientenalters erfolgt die Therapie je nach Allgemeinzustand, fitte Patienten erhalten Fludarabin/Cyclophosphamid/ Rituximab (FCR) [17], bei >65-jährigen fitten Patienten kann auch BendamustinRituximab eingesetzt werden [14].

Patienten mit relevanter Komorbidität oder reduziertem Allgemeinzustand können Chlorambucil/Obinutuzumab erhalten, wobei bisher nur ein Vorteil mit progressionsfreiem Überleben gezeigt werden konnte [23].

Bei komorbiden Patienten kann auch primär eine Therapie mit Ibrutinib durchgeführt werden [6]. Alternativ ist eine Therapie mit Idelalisib möglich, hier muss jedoch auf immunologisch vermittelte Diarrhö als Nebenwirkung und eine erhöhte Infektionsrate geachtet werden [19].

Bei Nachweis einer del17p/TP53-Mutation ist eine Chemotherapie nicht wirksam, und eine Therapie mit Ibrutinib oder Idelalisib/Rituximab wird als Ersttherapie empfohlen.

Die CLL kann mehrere Autoimmunphänomene auslösen wie eine Immunthrombopenie (ITP), eine Autoimmunhämolyse (AIHA) und eine Aplasie der erythroiden Hämatopoese („pure-red cell aplasia“ [PRCA]). Diese werden in der Regel mit Glukokortikoiden behandelt, ungeachtet der zugrunde liegenden CLL. Erst bei steroidresistentem Verlauf sollte eine CLL-spezifische Therapie erfolgen [14].

Neue Therapieansätze mit dem bcl2-Inhibitor Venetoclax [48] werden in 
Tab. 5 Altersabhängige Berechnung des Prognoseparameters für follikuläre Lymphome (FLIPI-Score)

\begin{tabular}{lll} 
& FLIPI 1 & FLIPI 2 \\
\hline Alter & $>60$ Jahre & $>60$ Jahre \\
Laktatdehydrogenase & $>$ Normalwert & - \\
\hline 2-Mikroglobulin & - & $>$ Normalwert \\
Lymphknoten & $>4$ Regionen & $>6 \mathrm{~cm}$ Diameter \\
\hline Knochenmarkbefall & - & Vorhanden \\
\hline Stadium & III/IV & - \\
$\mathrm{Hb}$ & $<12 \mathrm{~g} / \mathrm{dl}$ & $<12 \mathrm{~g} / \mathrm{dl}$
\end{tabular}

mehreren Studien getestet. Die optimale Sequenz der vielfältigen neuen Ansätze, ihr Stellenwert in Kombination oder anstelle der konventionellen Chemotherapie als auch die Rolle der allogenen Stammzelltransplantation bei rezidivierter CLL muss neu definiert werden.

Liegt keine Lymphozytose vor und der CLL-typische Immunphänotyp liegt im Lymphknoten vor, spricht man von kleinzelligem lymphozytischem Lymphom (SLL).

\section{Follikuläres Lymphom}

Das follikuläre Lymphom ist der typische Vertreter des indolenten NHL, die Inzidenz beträgt $3 / 100.000$. Auch hier fallen regelhaft Lymphknotenschwellungen auf, B-Symptome sind seltener als beim DLBCL, histologisch wird der Anteil von Blasten pro Gesichtsfeld quantifiziert und das follikuläre Lymphom graduiert (Grad 1: $<5 \%$, Grad 2: 5-10\%, Grad 3: $15 \%)$.

\section{\) Das follikuläre Lymphom ist der typische Vertreter des indolenten Non-Hodgkin- Lymphoms}

Zur Prognoseabschätzung wird der follikuläre Lymphom-IPI verwendet (- Tab. 5), der in 2 Versionen vorliegt [16, 54].

Limitierte Stadien I/II können kurativ mit einer $24 \mathrm{~Gy}$ Involved-field-Bestrahlung behandelt werden, eine zusätzliche Rituximab-Therapie erhöht vielleicht die Heilungschance und wird in einer Studie geprüft [62]. Fortgeschrittene Stadien werden erst in symptomatischen Stadien behandelt (Organkompression, Zyto- penie, B-Symptome, Ergussbildung), alle anderen können klinisch beobachtet werden („watch and wait“) [12].

Eine Chemotherapie wird in palliativer Intention durchgeführt, es besteht jedoch ein sehr langes progressionsfreies bzw. therapiefreies Intervall. Bendamustin alle 4 Wochen oder CHOP alle 3 Wochen in Kombination mit Rituximab, gefolgt von einer 2-jährigen Erhaltungstherapie mit Rituximab gilt als Standard [47].

Im Rezidiv muss zunächst nicht zwangsläufig behandelt werden, sondern analog der Primärtherapie eine entsprechende Symptomatik aufgetreten sein. Es sollte zunächst eine vermutlich nicht-kreuzresistente Chemotherapie + Rituximab eingesetzt werden. Bei jüngeren Patienten und kurzer Remission ist eine autologe Stammzelltransplantation, bei mehrfachem Rezidiv auch eine allogene Stammzelltransplantation indiziert. Oft kann bei begrenztem Rezidiv eine Strahlentherapie oder bei langem therapiefreiem Intervall die Ersttherapie wiederholt werden. Viele Chemotherapien sind wirksam, so können Gemcitabin/ Oxaliplatin oder Fludarabin-Kombinationen eingesetzt werden, wobei eine Langzeittoxizität insbesondere der Hämatopoese beachtet werden soll. Sehr wirksam ist auch eine Radioimmuntherapie mit ${ }^{90}$ Yttrium-IbritumomabTiuxetan (Zevalin@) [12].

Neue Ansätze beinhalten den optimierten CD20-Antikörper Obinutuzumab, der aktuell in einer großen PhaseIII-Studie GALLIUM getestet wird, nachdem er im Rezidiv bereits erfolgreich eingesetzt wurde [36].

Im Rezidiv ist der PI3-Kinase-Inhibitor Idelalisib zugelassen, wenn 2 Vortherapien erfolgt sind [22]. Der Bruton-
Tyrosinkinase-Inhibitor Ibrutinib [3] und der bcl-2-Inhibitor Venetoclax [56] könnten ebenfalls wirksam sein, eine Zulassung besteht jedoch nicht. Eine Kombination von Rituximab und Lenalidomid war sehr effektiv in einer PhaseII-Studie [35].

\section{Lymphome bei rheumatischen Erkrankungen}

Das Risiko für die Entwicklung eines Lymphoms ist bei verschiedenen entzündlichen Systemerkrankungen erhöht, auch wenn die in der Literatur berichteten Inzidenzraten variieren. $\mathrm{Zu}$ den Erkrankungen, die mit einer erhöhten Lymphominzidenz einhergehen, gehören vornehmlich die rheumatoide Arthritis (RA), das Sjögren-Syndrom, der systemische Lupus erythematodes (SLE), die Zöliakie sowie die Autoimmunthyreoiditis [53].

\section{》) Das Risiko für ein Lymphom ist bei verschiedenen entzünd- lichen Systemerkrankungen erhöht}

Daten aus Kohortenanalysen zu Patienten mit RA und Sjögren-Syndrom zeigen, dass die Lymphominzidenz mit der Schwere bzw. Aktivität der Erkrankung assoziiert ist [1]. Ein erhöhtes Risiko mit steigendem Alter und bei Männern wird - ähnlich wie in der Normalbevölkerung - auch bei RA-Patienten gesehen.

Die Gruppe der malignen Lymphome ist bezüglich Morphologie, klinischer Charakteristika sowie möglicher ätiopathogenetischer Faktoren heterogen. Eine gewebsspezifische chronische Antigenexposition scheint pathogenetisch von Bedeutung zu sein, Lymphommanifestationen finden sich insbesondere in den betroffenen Organen. Beispiele sind das mukosaassoziierte Lymphom (MALT) beim Sjögren-Syndrom in den Speicheldrüsen oder das dünndarmassoziierte T-Zell-Lymphom bei der Zöliakie.

Bei der RA bzw. dem SLE wird postuliert, dass die kontinuierliche Immunstimulation, insbesondere die der B-Zellen, ein Risikofaktor für eine Lymphoment- 
stehung ist. Eine Assoziation des follikulären Lymphoms mit dem Rauchen beim Vorliegen bestimmter HLA-DRB1-Varianten konnte kürzlich aufgezeigt werden [2].

Eine vor Kurzem erschienene $\mathrm{Pu}$ blikation aus Schweden untersucht die Assoziation zwischen verschiedenen Lymphomentitäten und Autoimmunerkrankungen. Interessanterweise zeigte sich eine Assoziation insbesondere mit den B-Zell-Lymphomen, deren Ursprung in den Keimzentren gesehen wird. Dazu gehören das diffus großzellige Lymphom, das follikuläre Lymphom und das Hodgkin-Lymphom. Lymphome mit Ursprung außerhalb der Keimzentren wie CLL, akute lymphatische Leukämie (ALL) oder Myelom zeigten nur vereinzelt eine Assoziation zu den untersuchten Autoimmunerkrankungen [28].

\section{Lymphomrisiko bei verschiedenen entzündlich rheumatischen Erkrankungen}

\section{Lymphome und rheumatoide Arthritis}

Daten aus dem britischen Register BSRBR zur Lymphominzidenz bei nicht mit Biologika behandelten RA-Patienten zeigen eine erhöhte Inzidenz für das NHL sowie das Hodgkin-Lymphom [38]. Die standardisierte Inzidenzrate (SIR) der NHL zeigte sich mit 3,81 (95\%-Konfidenzintervall [KI] 2,36-5,82) sowie des Hodgkin-Lymphoms mit einer SIR von 12,82 (95\%-KI 4,16-29,92) signifikant erhöht. Das DLBCL scheint dabei insbesondere mit der Krankheitsaktivität der RA assoziiert zu sein, nahezu $48 \%$ der beobachteten Lymphome zeigen diesen Subtyp [1]. Ein Zusammenhang mit der Einnahme verschiedener konventioneller Basistherapeutika konnte hingegen nicht gezeigt werden.

Im schwedischen Register wurden innerhalb einer Kohorte von 74.651 RAPatienten 378 Patienten mit Lymphom mit 378 gematchten Kontrollpatienten bezüglich ihrer Steroidtherapie verglichen. Dabei zeigte sich, dass die Einnahme von Glukokortikoiden über mehr als 2 Jahre mit einer Verminderung des Lymphomrisikos einherging (Odds Ratio [OR] 0,43, $95 \%$-KI 0,26-0,72), keinen Einfluss hatte die Dauer der RA vor Beginn der Steroidtherapie. In dieser Kohorte zeigte sich ein vermindertes Risiko für den DLBCL-Subtyp [25].

Die Gründe für die zu beobachtende erhöhte Inzidenz von Lymphomen bei der RA sind unklar. Diskutiert wird einerseits eine gemeinsame genetische Prädisposition, jedoch zeigen nahe Verwandte ebenso wie RA-Patienten vor der Diagnose keine erhöhte Lymphomrate. Trotz konsequenterem Einsatz von Immunsuppressiva und Biologika ist die Inzidenzrate der Lymphome nicht gestiegen. Die wahrscheinlichste Ursache ist daher eine gemeinsame Pathobiologie von Lymphomen und Autoimmunerkrankungen, insbesondere bei Patienten mit hoher Krankheitsaktivität. Die CLL scheint zu den Lymphomentitäten zu gehören, die zumindest bei der RA nicht gehäuft beobachtet werden. Neuere Daten aus dem schwedischen Register legen nahe, dass sich die Lymphomentitäten im Laufe der letzten Jahre verändert haben. So war früher der Anteil von RA-Patienten, die ein DLBCL entwickelten, höher als in der Normalbevölkerung. Aktuelle Daten zeigen einen rückläufigen Anteil von Patienten mit DLBCL, hingegen eine relative Zunahme des Anteils von RA-Patienten mit Hodgkin- bzw. Mantelzelllymphom [26].

\section{Lymphome und Sjögren-Syndrom}

Unter den entzündlichen Systemerkrankungen haben Patienten mit einem primären Sjögren-Syndrom das höchste Risiko, ein Lymphom zu entwickeln. Das Risiko ist 7- bis 19-fach gegenüber der Normalbevölkerung erhöht. Das Risiko für die Entwicklung eines Lymphoms steigt mit zunehmender Krankheitsdauer und zeigt ein kumulatives Risiko von $3,4 \%$ nach 5 Jahren und 9,8\% nach 15 Jahren [55].

Eine französische multizentrische Studie untersucht die Frage nach prädiktiven Faktoren für die Entwicklung eines Lymphoms mithilfe einer FallKontroll-Studie. Unter den 101 Patienten mit Sjögren-Syndrom und Lymphom waren mehrheitlich Frauen $(86,1 \%)$ mit einem mittleren Alter von 57,4 $( \pm 12,6)$ Jahren zum Zeitpunkt der Diagnose. Die Lymphome waren fast ausschließlich
NHL, mehrheitlich Marginalzonenlymphome $(76,8 \%)$ mit Beteiligung des mukosaassoziierten Gewebes im Sinne eines MALT-Lymphoms (58,6\%), das zumeist in den Speicheldrüsen lokalisiert war. Andere mögliche Manifestationsorte sind Orbita, Nasopharynx, Magen, Schilddrüse und Lunge [42]. In einer multivariaten Analyse zeigten sich die klinische Vergrößerung der Speicheldrüsen, der Nachweis von Rheumafaktoren, niedrigem C4, Kryoglobulinen, einer Lymphopenie sowie höhere Krankheitsaktivität (ESSDAI $\geq 5$ ) als Risikofaktoren für eine Lymphomentwicklung. Eine andere Studie konnte zusätzlich den Nachweis von Anti-Ro und/oder AntiLa und den Nachweis einer monoklonalen Gammopathie als unabhängigen Risikofaktor für die Entwicklung eines Lymphoms charakterisieren [18].

\section{》) Bei entzündlichen Systemerkrankungen besteht beim primären Sjögren-Syndrom das höchste Lymphomrisiko}

Pathophysiologisch wird ein mehrstufiger Prozess in der Lymphomgenese postuliert (-Abb. 3). Die chronische Antigenstimulation einschließlich Stimulation einer B-Zell-Antwort gegen Autoantigene (Ro und La) in ektopischen Keimzentren in den Speicheldrüsen führt zur Bildung von Immunkomplexen. Diese wiederum aktivieren polyklonale B-Zellen innerhalb der Marginalzone, wobei die Entwicklung einer Monoklonalität bzw. eines Lymphoms durch eine parakrine/autokrine Wirkung von BAFF, Mutationen von Onkogenen und eine Überaktivierung des NF-kB-Signalweges potenziell akzeleriert werden kann.

Der Verlauf von Sjögren-assoziierten Non-Hodgkin-Lymphomen ist typischerweise indolent, die geringe $\mathrm{Tu}$ morlast korreliert zu einer meist normwertigen Laktatdehydrogenase (LDH) und $\beta 2$-Mikroglobulin. B-Symptome fehlen in der Regel, und eine Knochenmarkbeteiligung ist selten. Bei $10 \%$ der Patienten kann es zu einer Transformati- 


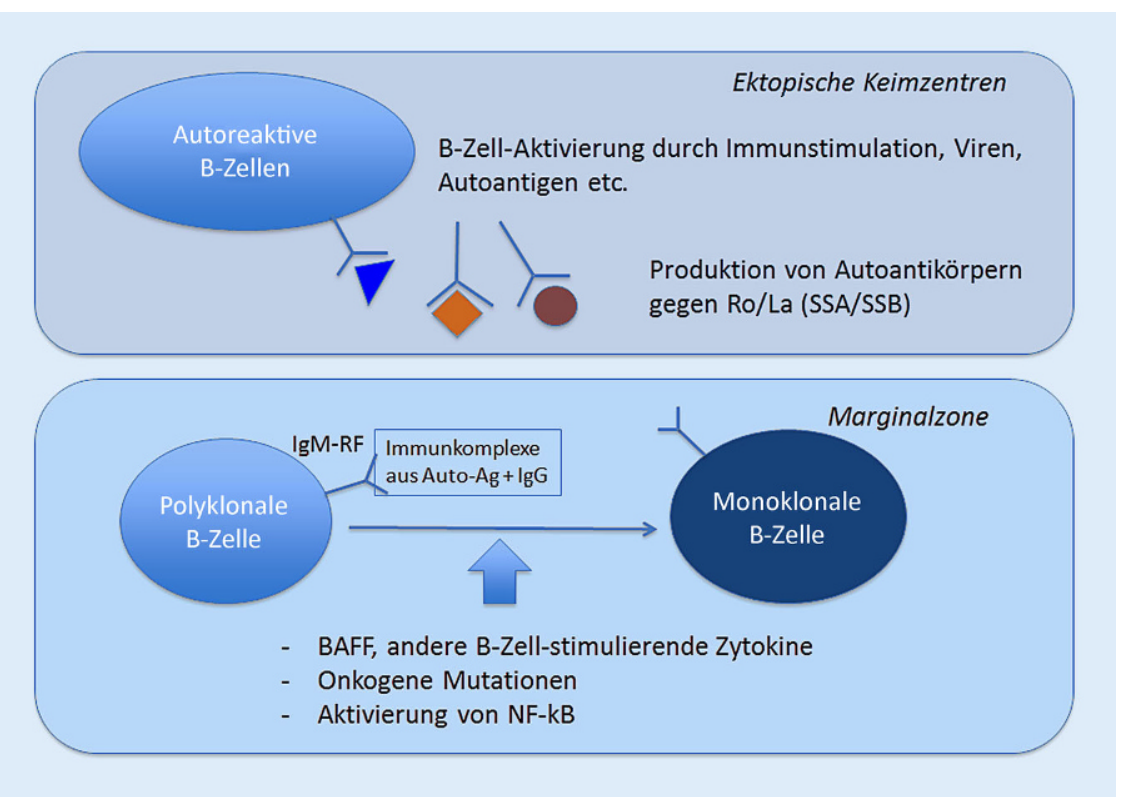

Abb. 3 ॥ Mehrstufiges Modell zurLymphomentstehung beim Sjögren-Syndrom.SSA/SSB, IgM-RFlgM Rheumafaktoren, Auto-Ag Autoantigen, IgG Immunglobulin G, BAFF. (Adaptiert nach [41])

on in ein diffus großzelliges B-NHL mit aggressiverem Verlauf kommen [41].

Damit ist die Prognose des Sjögrenassoziierten NHL insgesamt gut. Eine retrospektive Studie an 53 Patienten zeigt ein 3-Jahres-Überleben von $97 \%$ sowie ein ereignisfreies Überleben (EFS) von $78 \%[61]$.

\section{Lymphome bei anderen Kollagenosen}

In einer Metaanalyse, basierend auf 20 Studien, zeigte sich neben dem signifikant erhöhten Risiko für die Entwicklung eines NHL bei Patienten mit primärem Sjögren-(SIR 18,8, 95 \%-KI 9,5-37,3) ein erhöhtes Risiko für Patienten mit SLE (SIR 7,4; 95\%-KI 3,3-17) [65].

Aktuelle Daten aus den schwedischen Registern zeigen auch bei der Sklerodermie eine erhöhte SIR für das DLBCL (SIR 3,0 [95\%-KI 1,4-5,5]). Auch beim SLE zeigt das Register eine erhöhte SIR für das DLBCL (SIR 6,6 [95\%-KI 4,4-9,6]), das follikuläre Lymphom (SIR 3,0 [95\%-KI 1,4-5,7]) sowie das Hodgkin-Lymphom (SIR 8,4 [95\%-KI 5,2-12,9]). Keine erhöhte SIR fand sich hingegen beim diskoiden Lupus. Bei den Myositiden (Polyund Dermatomyositis) fand sich ebenfalls eine erhöhte SIR für das DLBCL (SIR 5,8 [95\%-KI 2,6-11]), für das follikuläre Lymphom (SIR 5,1 [95\%-KI 1,6-12])

\section{Lymphomrisiko unter DMARDs und Biologika}

\section{Lymphomrisiko unter konventio- nellen DMARDs}

Azathioprin kann das Risiko für lymphoproliferative Erkrankungen erhöhen, jedoch wird andererseits Azathioprin häufiger bei Patienten mit höherer Krankheitsaktivität bei entzündlichen Systemerkrankungen eingesetzt, seltener bei der RA mit alleiniger Gelenkmanifestation.

Ältere schwedische Daten konnten neben der Assoziation einer erhöhten Lymphominzidenz mit höherer Krankheitsaktivität lediglich eine statistisch leicht erhöhte Lymphominzidenz unter Azathioprin, nicht aber unter intramuskulärem Gold, Antimalariamittel, Sulfasalazin oder Methotrexat (MTX) zeigen [1].

Hepatosplenische T-Zell-Lymphome mit ungünstigem Verlauf sind selten und wurden früher hauptsächlich bei jungen Männern mit entzündlichen Darmerkrankungen berichtet. Eine Analyse basierend auf FDA (Food and Drug Administration)-Daten umfasst 91 Fälle mit T-Zell-Lymphom (mehrheitlich hepatosplenische T-Zell-Lymphome) aus Meldungen und 9 zusätzliche publizierte Fälle. In der Kohorte waren mehrheitlich Patienten mit RA bzw. Morbus Crohn, aber auch Patienten mit Psoriasis, Colitis ulcerosa und ankylosierender Spondylitis; $68 \%$ der Patienten hatten eine Therapie mit TNF-Blockern in Kombination mit Immunmodulatoren. Das Risiko eines T-Zell-Lymphoms war höher bei TNFBlockern in Kombination mit Thiopurinen (95\%-KI 4,98-354,09, $p<0,0001$ ) und Thiopurinen allein als unter TNFBlocker-Therapie ohne Thiopurine [11]. Eine ältere Metaanalyse, basierend auf 26 Studien und 8905 Morbus-Crohn-Patienten bzw. 21.178 Patientenjahren berichtet von insgesamt 13 NHL-Fällen und somit einer sehr niedrigen Inzidenz von 6,1/10.000 Patientenjahren, die meisten hatten eine Therapie mit TNF-Blockern und Immunmodulatoren [50].

Verschiedene Register zeigen, dass RA-Patienten unter MTX im Vergleich zur gesunden Normalbevölkerung eine erhöhte Lymphomrate haben. Diese scheint jedoch die mit der RA assoziierte erhöhte Lymphominzidenz widerzu- 
spiegeln und nicht durch MTX bedingt $\mathrm{zu}$ sein. Andererseits wurde in asiatischen Patientenkollektiven mehrfach über das Auftreten von MTX-assoziierten lymphoproliferativen Erkrankungen (MTX-LPD) berichtet, die im Verlauf nach Absetzen des MTX bei einem relevanten Anteil der Patienten spontan regredient waren [29]. Zumindest bei einem Teil der MTX-LPD-Patienten wurde eine EBV (Epstein-Barr-Virus)Reaktivierung unter MTX postuliert. Eine Regression der MTX-LPD wurde nicht selten bei einer Erholung der peripheren Lymphozytenzahlen beobachtet. Die meisten Berichte von möglichen MTX-LPD kommen interessanterweise aus dem asiatischen Raum, sodass die Ethnizität der Patienten von Bedeutung sein könnte.

\section{\) Bei einem Teil der MTX- LPD-Patienten wurde eine EBV-Reaktivierung unter MTX postuliert}

Die Entscheidung, ob sofort nach Diagnose eines Lymphoms mit einer Polychemotherapie begonnen werden muss oder ob zunächst abgewartet werden kann, muss daher individuell getroffen werden und hängt maßgeblich von Stadium und Aggressivität des Lymphoms sowie der EBV-Positivität ab.

\section{Lymphomrisiko und Biologika}

TNF-Blocker. Die Diskussion, ob TNFBlocker mit einer erhöhten Lymphominzidenz assoziiert sein könnten, basiert im Wesentlichen auf einer Metaanalyse aus dem Jahr 2006, die 9 randomisiert kontrollierte Studien mit insgesamt 3493 RAPatienten unter Infliximab bzw. Adalimumab im Hinblick auf schwere Infektionen und Malignominzidenz analysierte. Dabei zeigte sich eine höhere Malignombzw. Lymphominzidenz bei den höheren Dosierungen innerhalb von klinischen Studien, nicht aber bei den heute zugelassenen Dosierungen [5]. Ein weiterer Kritikpunkt dieser Analyse ist die fehlende Berücksichtigung von Etanercept und eine unerwartet niedrige Ereignisrate bei den Kontrollpatienten. Bezüglich der Dosis der TNF-Blocker zeigt jedoch auch die Sicherheitsdatenbank von Golimumab über 5 Jahre eine numerisch höhere Inzidenz von Lymphomen unter der 100 mg/Monat-Dosierung [32].

Daten aus dem britischen Register, basierend auf mehr als $120.000 \mathrm{~Pa}$ tientenjahren unter TNF Inhibitoren (TNFi) oder konventioneller Basistherapie, konnten keinen Hinweis für eine erhöhte Lymphominzidenz unter TNFi im Vergleich zu Patienten unter konventioneller Basistherapie erbringen [39]. Auch eine zuvor publizierte Metaanalyse, basierend auf einer systematischen Literatursuche, zeigte keine Risikoerhöhung unter TNF-Blockern für Lymphome [37].

Eine aktuelle Analyse aus dem schwedischen Register unter Einschluss von RA-Patienten zwischen 1997 und 2012 bestätigt das erhöhte Lymphomrisiko bei der RA mit einer Hazard Ratio (HR) von 1,6 (95\%-KI 1,2-2,1). Eine Assoziation zum Jahr der Diagnose, zur Aktivität der Erkrankung im ersten Jahr oder zum Einsatz von MTX bzw. TNF-Blockern wurde nicht beobachtet, hingegen waren Glukokortikoide im ersten Jahr mit einer Risikoverminderung assoziiert (HR 0,5; $95 \%$-KI 0,3-0,9). Statistisch signifikant häufiger traten Lymphome nach mehr als 6 Jahren Krankheitsdauer auf, eine CLL wurde seltener, ein Hodgkin- und Mantelzelllymphom wurden jedoch häufiger als in der Allgemeinbevölkerung beobachtet [26].

\section{) Statistisch signifikant häufiger traten Lymphome nach mehr als 6 Jahren Krankheitsdauer auf}

Letztlich bleiben jedoch Fragen zur genauen Rolle von TNF-Blockern in diesem Kontext offen. Einerseits wäre davon auszugehen, dass eine langfristig verbesserte Krankheitsaktivität mit einer Reduktion der Lymphominzidenz assoziiert ist. Andererseits werden insbesondere Patienten mit schwerer Erkrankung mit Biologika behandelt. Eine längerfristige Immunsuppression könnte ihrerseits zu Störungen der Immunüberwachung führen, was in der Folge wiederum die Entstehung von Lymphomen begünstigen könnte. Diese Überlegungen unterstreichen die Bedeutung von Registerdaten, die den Verlauf von Patienten unter TNFi und anderen Biologika dokumentieren.

Unklar bleibt weiterhin, ob es Unterschiede zwischen den einzelnen TNFBlockern gibt, insbesondere dem löslichen Rezeptor und den monoklonalen Antikörpern. Beide neutralisieren lösliches TNF alpha (TNFa), hingegen binden nur die Antikörper an membrangebundenes TNFa. Diese Unterschiede im Wirkmechanismus werden zur Erklärung des unterschiedlichen Wirkprofils bei bestimmten entzündlichen Entitäten wie der Uveitis oder bei den entzündlichen Darmerkrankungen herangezogen. Daten aus dem französischen RATIORegister bei allerdings nur 38 Lymphomen (31 NHL, 7 Hodgkin) zeigten für Patienten unter Adalimumab oder Infliximab ein höheres Lymphomrisiko im Vergleich zu Etanercept (SIR 4,1 [95\%KI 2,3-7,1] bzw. 3,6 [95\%-KI 2,3-5,6] vs. 0,9 [95\%-KI 0,4-1,8]) [37]. Diese Unterschiede zwischen den einzelnen TNFBlockern werden jedoch von den anderen großen europäischen Registern nicht bestätigt. Hingegen bestätigt die Analyse des BSRBR die Prädominanz des DLBCL unter den beobachteten Lymphomsubtypen [39]. Auch wenn dies nicht statistisch signifikant war, zeigte sich das DLBCL mit einer HR von 1,54 (95\%KI 0,60-3,95) numerisch häufiger unter TNF-Blockern im Vergleich zu konventionellen DMARDs [43]. In den aktuellen schwedischen Registerdaten findet sich der Anteil des DLBCL rückläufig im Vergleich zu den historischen RA-Kohorten und entspricht im Wesentlichen der Normalbevölkerung [26].

Gegen eine TNFi-assoziierte Lymphominduktion sprechen auch Daten aus den Registern, die die Inzidenz von Malignomen unter TNFi-behandelten Patienten mit ankylosierender Spondylitis und Psoriasisarthritis untersuchen. Dabei zeigte sich in einer Analyse von ARTIS und DANBIO keine erhöhte Lymphominzidenz unter TNFi bei diesen Patienten. Für Patienten mit Psoriasisarthritis unter MTX und/oder 
Sulfasalazin zeigte sich ein marginales Lymphomrisiko mit einer HR von 1,7 (95\%-KI 1,0-3,1) [27]. Zu Adalimumab wurden Daten zur Malignominzidenz bei Patienten mit RA, ankylosierende Spondylitis (AS), Psoriasisarthritis, Psoriasis und Morbus Crohn verglichen. Dabei zeigte sich nur für RA-Patienten eine erhöhte SIR für Lymphome [7].

\section{Lymphominzidenz bei nicht TNF blockierenden Biologika}

Tocilizumab und Lymphome. In den $\mathrm{Zu}$ lassungsstudien bzw. ihrer Langzeitverlängerung an mehr als 4000 RA-Patienten und einer Beobachtungszeit von mehr als 16.000 Patientenjahren zeigte sich für die USA eine nicht erhöhte SIR für Lymphome, marginal war diese für Patienten außerhalb der USA erhöht [46]. In der japanischen Postmarketing Surveillance wurden bei mehr als 5500 RA-Patienten insgesamt 13 maligne Lymphome beobachtet, damit zeigte sich die SIR insgesamt mit 3,13 (95 \%-KI 1,82-5,39) erhöht, betroffen waren insbesondere Frauen $(n=$ 4542) mit einer SIR von 3,89 (95\%-KI 2,09-7,22) [64].

Abatacept und Lymphome. In der Sicherheitsdatenbank zu Abatacept aus 7 klinischen Studien und mehr als 4000 Patienten zeigte sich keine erhöhte Lymphominzidenz unter Abatacept im Vergleich zur Normalbevölkerung [52]. Im Langzeitverlauf zeigte sich die Lymphominzidenz tendenziell höher unter Abatacept im Vergleich zur US-Normalbevölkerung, jedoch vergleichbar zu Daten aus RA-Kohorten [63].

Rituximab und Lymphome. Rituximab ist als Anti-CD20-gerichteter monoklonaler Antikörper Bestandteil vieler Therapieregime bei verschiedenen Lymphomentitäten (s.oben). Die Sicherheitsdaten der Longterm-ExtensionStudie des Phase-3-Studienprogrammes zeigen keine erhöhte Lymphominzidenz [59]. Daten aus dem deutschen RABBITRegister zeigen, dass bei vorbestehendem Lymphom und Notwendigkeit einer Intensivierung der antirheumatischen Therapie von den am Register teilnehmenden Rheumatologen bevorzugt Ri- tuximab bzw. konventionelle DMARDs eingesetzt werden [57].

JAK-Inhibitoren und Lymphome. Innerhalb des weltweiten Tofacitinib-Studienprogrammes wurden bei 5671 RA-Patienten insgesamt 10 Lymphome beobachtet, es zeigte sich im Weiteren kein $\mathrm{Zu}$ sammenhang mit Dosis bzw. Dauer der Therapie. Histopathologisch waren diese Lymphome heterogen, bei einem Patienten zeigte sich eine EBV-Reaktivierung [10]. Daten zum Baricitinib-Studienprogramm zur Frage der Lymphominzidenz liegen aktuell noch nicht vor.

\section{Fazit für die Praxis}

- Die Inzidenz von Lymphomen, insbesondere $\mathrm{NHL}$, ist bei verschiedenen rheumatischen Erkrankungen, insbesondere beim Sjögren-Syndrom und der RA, gegenüber der gesunden Bevölkerung erhöht.

- Daher sollte bei klinischem Verdacht eine entsprechende Diagnostik eingeleitet werden.

- Insbesondere bei Patienten mit hoher Krankheitsaktivität ist das Lymphomrisiko erhöht.

- Der Verlauf der Sjögren-assoziierten MALT-Lymphome ist relativ indolent, hingegen gehört bei der RA das DLBCL neben dem follikulären Lymphom zu den häufigsten Lymphomentitäten.

- Unter einer Rituximab enthaltenden Chemotherapie ist in der Regel eine spezifische immunsupprimierende Therapie nicht erforderlich, nach Abschluss der Lymphomtherapie werden konventionelle DMARDs bzw. Rituximab als antirheumatische Therapie bevorzugt.

- Zur Frage eines erhöhten Lymphomrisikos unter Biologikatherapie stehen keine Daten aus randomisierten klinischen Studien zur Verfügung. Die vorhandenen Registerdaten zeigen jedoch, dass kein zusätzliches Risiko, bedingt durch den Einsatz eines Biologikums, beobachtet wurde.

\section{Korrespondenzadresse}

Prof. Dr. A. Rubbert-Roth

Medizinische Klinik I der Universität Köln

Joseph-Stelzmann Str. 9, 50924 Köln,

Deutschland

andrea.rubbert@uk-koeln.de

\section{Einhaltung ethischer Richtlinien}

Interessenkonflikt. Dieses Manuskript entstand auf Basis einer von Roche und Chugai unterstützten Arbeitsgruppe, aber ohne jegliche Einflussnahme der Firmen auf das Ergebnis oder die Publikation. Der Beitrag ist Teil eines Supplements gesponsert von Chugai/Roche. A. Rubbert-Roth, J.T. Bittenbring und $\mathrm{G}$. Assmann geben an, dass kein Interessenkonflikt besteht.

Dieser Beitrag beinhaltet keine von den Autoren durchgeführten Studien an Menschen oder Tieren.

\section{Literatur}

1. Baecklund E, Iliadou A, Askling J et al (2006) Association of chronic inflammation, not ist treatment, with increased lymphoma risk in rheumatoid arthritis. Arthritis Rheum 54(3):692-701

2. Baecklund F, Foo JN, Askling J et al (2017) Possible interaction between cigarette smoking and HLADRB1 variation in the risk of follicular lymphoma. Am J Epidemiol 185(8):681-687

3. Bartlett NL et al (2014) Ibrutinib Monotherapy in Relapsed/Refractory Follicular Lymphoma (FL): Preliminary Results of a Phase 2 Consortium (P2C) Trial. Blood 124:LP800

4. Binet JLetal (1981) A new prognostic classification of chronic lymphocytic leukemia derived from a multivariate survival analysis. Cancer 48:198-206

5. Bongartz T, Sutton AJ, Sweeting MJ et al (2006) Anti TNF antobidy therapy in rheumatoid arthritis and the risk of serious infections and malignancies. JAMA 295:2275-2285

6. Burger JA et al (2015) Ibrutinib as Initial Therapy for Patients with Chronic Lymphocytic Leukemia. N Engl J Med. https://doi.org/10.1056/ NEJMoa1509388

7. Burmester GR, Panaccione R, Gordon KB et al (2013) Adalimumab: longterm safety in 23458 patients from global clinical trials in rheumatoid arthritis, juvenile idiopathic arthritis, ankylosing spondylitis, psoriatic arthritis, psoriasis and Crohn's disease. Ann Rheum Dis 72(4):517-524

8. Carbone PP, Kaplan HS, Musshoff K, Smithers DW, Tubiana M (1971) Report of the Committee on Hodgkin â€ $\epsilon^{T M}$ s Disease Staging Classification. Cancer Res 31:1860-1861

9. Crump M et al (2014) Randomized comparison of gemcitabine, dexamethasone, and cisplatin versus dexamethasone, cytarabine, and cisplatin chemotherapy before autologous stem-cell transplantation for relapsed and refractory aggressive lymphomas: NCIC-CTG LY.12. J Clin Oncol.https://doi.org/10.1200/JCO.2013.53.9593

10. Curtis JR, Lee EB, Kaplan IV et al (2016) Tofacitinib, an oral Janus kinase inhibitor: analysis of malignancies across the rheumatoid arthritis clinical development program. Ann Rheum Dis 75((5):831-841 
11. Deepak P, al Sifuentes Sherid HM et al (2013) T cell non-Hodgkin's lymphoma reported tot he FDA AERS with tumor necrosis factor-alpha (TNFa) inhibitors: Results of the REFURBISH study. Am J Gastroenterol 108((1):99-105

12. Dreyling $M$ et al (2016) Newly diagnosed and relapsed follicular lymphoma: ESMO clinical practice guidelines for diagnosis, treatment and follow-up. Ann Oncol 27(v83):v90

13. Eichenauer DA et al (2014) Hodgkin's lymphoma: ESMO clinical practice guidelines for diagnosis, treatment and follow-up. Ann Oncol 25(iii)70):iii75

14. Eichhorst B et al (2015) Chronic lymphocytic leukaemia: ESMO Clinical Practice Guidelines for diagnosis, treatment and follow-up. Ann Oncol. https://doi.org/10.1093/annonc/mdv303

15. Engert A et al (2012) Reduced-intensity chemotherapy and PET-guided radiotherapy in patients with advanced stage Hodgkin's lymphoma (HD15 trial): a randomised, open-label, phase 3 noninferiority trial. Lancet 379:1791-1799

16. Federico $M$ et al (2009) Follicular lymphoma international prognostic index 2:A new prognostic index for follicular lymphoma developed by the international follicular lymphoma prognostic factor project. J Clin Oncol. https://doi.org/10. 1200/JCO.2008.21.3991

17. Fischer $\mathrm{K}$ et al (2016) Long-term remissions after FCR chemoimmunotherapy in previously untreated patients with CLL:Updated results of the CLL8 trial. Blood 127:208-215

18. Fragkioudaki S, Mavragani CP, Moutsopoulos HM (2016) Predicting the risk for lymphoma development in Sjogren syndrome. Medicine (Baltimore) 95(25):e3766

19. Furman RR et al (2014) Idelalisib and Rituximab in relapsed chronic lymphocytic leukemia. N Engl J Med.https://doi.org/10.1056/NEJMoa1315226

20. Gisselbrecht C et al (2010) Salvage regimens with autologous transplantation for relapsed large B-cell lymphoma in the rituximab era. J Clin Oncol 28:4184-4190

21. Glass B et al (2014) Rituximab after lymphomadirected conditioning and allogeneic stemcell transplantation for relapsed and refractory aggressive non-Hodgkin lymphoma (DSHNHL R3): An open-label, randomised, phase 2 trial. Lancet Oncol. https://doi.org/10.1016/S14702045(14)70161-5

22. Gopal AK et al (2014) PI3Kס inhibition by Idelalisib in patients with relapsed indolent lymphoma. NEngl J Med 370:1008-1018

23. Goede V et al (2014) Obinutuzumab plus Chlorambucil in patients with CLL and coexisting conditions. NEngl J Med 370:1101-1110

24. Hallek M et al (2008) Guidelines for the diagnosis and treatment of chronic lymphocytic leukemia: A reportfrom the International Workshopon Chronic Lymphocytic Leukemia updating the National Cancer Institute-Working Group 1996 guidelines. Blood. https://doi.org/10.1182/blood-2007-06093906

25. Hellgren K, lliadou A, Rosenquist R et al (2010) Rheumatoid arthritis, treatment with corticosteroids and risk of malignat lymphomas: results from a case-control study. Ann Rheum Dis 69((4):654-659

26. Hellgren K, Baecklund E, Backlin C et al (2017) Rheumatoid arthritis and risk of lymphoma-is the risk still increased? Arthritis Rheumatol 69:700-708

27. Hellgren K, Dreyer L, Arkema EV et al (2017) Cancer risk in patients with spondylosarthritis treated with TNF inhibitors: a collaborative study from the ARTIS and DANBIO registers. Ann Rheum Dis 76((1):105-111

28. Hemminki K, Liu X, Ji J et al (2016) Origin of B cell neoplasms in autoimmune disease. PLOS ONE 11(6):e158360

29. Inui Y, Matsuoka H, Yakushijn K et al (2015) Methotrexate-associated lymphoproliferative disorders: management by watchful waiting and observation of early lymphocyte recovery after methotrexate withdrawal. Leuk Lymphoma 56 811:3045-3051

30. International CLL-IPI working group (2016) T. An international prognostic index for patients with chronic lymphocytic leukaemia (CLL-IPI): a metaanalysis of individual patient data. Lancet Oncol 17:779-790

31. Jaeger $U$ et al (2015) Rituximab maintenance for patients with aggressive B-cell lymphoma in first remission: Results of the randomized NHL13 trial. Haematologica. https://doi.org/10.3324/ haematol.2015.125344

32. Kay J, Fleischmann R, Keystone E et al (2016) Five-year safety data from 5 clinical trials of subcutaneous golimumab in patients with rheumatoid arthritis, psoriatic arthritis and ankylosing spondylitis. J Rheumatol 43:2120-2130

33. Kochenderfer JN et al (2015) Chemotherapyrefractory diffuse large B-cell lymphoma and indolent B-cell malignancies can be effectively treated with autologous $T$ cells expressing an anti-CD19 chimeric antigen receptor. J Clin Oncol. https://doi.org/10.1200/JCO.2014.56.2025

34. Lenz G et al (2008) Stromal Gene Signatures in Large-B-Cell Lymphomas. N Engl J Med. https:// doi.org/10.1056/NEJMoa0802885

35. Leonard JP et al (2015) Randomized trial of lenalidomide alone versus lenalidomide plus rituximab in patients with recurrent follicular lymphoma: CALGB 50401 (Alliance). J Clin Oncol. https://doi.org/10.1200/JCO.2014.59.9258

36. Marcus RE, Davies AJ, Ando $K$ et al (2016) Obinutuzumab-Based Induction and Maintenance Prolongs Progression-Free Survival (PFS) in Patients with Previously Untreated Follicular Lymphoma: Primary Results of the Randomized Phase 3 GALLIUM Study. ASH, San Diego (Abstract 6)

37. Mariette X, Gottenberg JE, Ravaud P et al (2011) Registries in rheumatoid arthritis and autoimmune diseases: data from the French registries. Rheumatology 50((1):222-229

38. Mercer LK, Davies R, Galloway JB et al (2013) Risk of cancer in patients receiving non-biologic diseasemodifying therapy for rheumatoid arthritis compared with the UK general population. Rheumatology 52:91-98

39. Mercer LK, Galloway JB, Lunt M et al (2016) Risk of lymphoma in patients exposed to antituomou necrosis factor therapy: results from the British Society for Rheumatology Biologics Register for Rheumatoid Arthritis. Ann Rheum Dis 76:497-503

40. Moskowitz CH et al (2015) Brentuximab vedotin as consolidation therapy after autologous stemcell transplantation in patients with Hodgkin's lymphoma at risk of relapse or progression (AETHERA): a randomised, double-blind, placebo controlled, phase 3 trial. Lancet 385:1853-1862

41. Nocturne G, Mariette X (2015) Sjögren syndromeassociated lymphomas: an update on pathogenesis and management. British J Haematol 168:317-327

42. Nocturne G, Virone A, Ng WF et al (2016) Rheumatoid factor and disease activity are independent predictors of lymphoma in primary Sjögren's syndrome. Arthritis Rheumatol 68((4):977-985

43. Nocturne G, Seror R, Mariette X (2017) Does the risk of lymphoma in patients with RA treated with TNF inhibitors differ according tot he histological subtype and the type of TNF inhibitor? Ann Rheum Dis 76(2):e3

44. Pfreundschuh Metal (2008) Six versus eight cycles of bi-weekly CHOP-14 with or without rituximab in elderly patients with aggressive CD20+ B-cell lymphomas: a randomised controlled trial (RICOVER-60). Lancet Oncol 9:105-116

45. Pfreundschuh $M$ et al (2014) Optimization of rituximab for the treatment of diffuse large B-cell lymphoma (II): Extended rituximab exposure time in the SMARTE-R-CHOP-14 trial of the German high-grade non-Hodgkin lymphoma study group. JClin Oncol 32:4127-4133

46. Rubbert-Roth A, Sebba A, Brockwell L et al (2016) Malignancy rate in patients with rheumatoid arthritis treated with tocilizumab. RMD Open 2(1):e213

47. Salles $\mathrm{G}$ et al (2011) Rituximab maintenance for 2 years in patients with high tumour burden follicular lymphoma responding to rituximab plus chemotherapy (PRIMA): A phase 3, randomised controlled trial. Lancet 377:42-51

48. Seymour JF et al (2017) Venetoclax plus rituximab in relapsed or refractory chronic lymphocytic leukaemia: a phase 1b study. Lancet Oncol 18:230-240

49. Shang $W$, Ning $Y, X u X$ et al (2015) Incidence of cancer in ANCA-associated vasculitis: A metaanalysis of observational studies. PLOS ONE 10(5):e126016 (epublished). https:doi.org/10. 1371/journal.pone.0126016

50. Siegel CA, Marden SM, Persing SM et al (2009) Risk of lymphoma associated with combination anti-tumor necrosis factor and immunomodulator therapy fort he treatment of Crohn's disease: a meta-analysis. Clin Gastroenterol Hepatol 7:874-881

51. Simon H (1993) The New England Journal of Medicine Bd. 1993. Medical Society, Massachusetts (No other uses without permission. Copyright)

52. Simon TA, Smitten AL, Franklin J et al (2009) Malignancies in the rheumatoid arthritis abatacept clinical development programme: an epidemiological assessment. Ann Rheum Dis 68(12):1819-1826

53. Smedby K, Vajdic CM, Falster M et al (2008) Autoimmune disorders and risk of non-Hodgkin lymphoma subtypes: a pooled analysis within the InterLymph Consortium. Blood 111(8):4029-4038

54. Solal-Céligny P et al (2004) Follicular lymphoma international prognostic index. Blood. https://doi. org/10.1182/blood-2003-12-4434

55. Solans-Laque R, Lopez-Hernandez A, BoschGil JA et al (2011) Risk, prediction and clinical characteristics of lymphoma development in primary Sjögren's syndrome. Semin Arthritis Rheum 41:415-423

56. Stilgenbauer S et al (2016) Venetoclax in relapsed or refractory chronic lymphocytic leukaemia with 17p deletion: a multicentre, open-label, phase 2 study. Lancet Oncol. https://doi.org/10.1016/ S1470-2045(16)30019-5

57. Strangfeld A, Pattloch D, Herzer P, Edelmann E, Zinke S, Aringer M et al (2013) Risk Of Cancer Recurrence Or New Tumors In RA Patients With Prior Malignancies Treated With Various Biologic Agents. [abstract]. Arthritis Rheum 2013;65 Suppl 10:806. https://doi.org/10.1002/art.2013. 65.issue-s 10 


\section{Lymphome}

58. Tilly $\mathrm{H}$ et al (2015) Diffuse large B-cell lymphoma (DLBCL): ESMO Clinical Practice Guidelines for diagnosis, treatment and follow-up. Ann Oncol 26(v116):v125

59. Van Vollenhoven RF, Emery P, Bingham CO et al (2013) Longterm safety of rituximab in rheumatoid arthritis: 9.5 year follow-up of the global clinical trial programme with a focus on adverse events of interest in RA patients. Ann Rheum Dis 72:1496-1502

60. Viviani $S$ et al (2011) ABVD versus BEACOPP for Hodgkin's Lymphoma When High-Dose Salvage Is Planned. N Engl J Med 365:203-212

61. Voulgaris M, Ziakas PD, Papageorgiou A et al (2012) Prognosis and outcome of non-Hodgkin lymphoma in primary Sjogren syndrome. Medicine (Baltimore) 91:1-9

62. Witzens-Harig $M$, Hensel $M$, Unterhalt $M$, Herfarth K (2011) Treatment of limited stage follicular lymphoma with Rituximab immunotherapy and involved field radiotherapy in a prospective multicenter Phase II trial-MIR trial. BMC Cancer. https://doi.org/10.1186/1471-2407-11-87

63. Weinblatt ME, Moreland LW, Westhovens R et al (2013) Safety of abatacept administered intravenously in treatment of rheumatoid arthritis: Integrated analyses of up to 8 years of treatment from the abatacept clinical trial program. JRheumatol 40:787-797

64. Yamamoto K, Goto H, Hirao K et al (2015) Longterm safety of tocilizumab: Results from 3 years of followup postmarketing surveillance of 5573 patients with rheumatoid arthritis in Japan. JRheumatol 42((8):1368-1375

65. Zintzaras E, Voulgarelis M, Moutsopoulos HM (2005) The risk of lymphoma developemnt in autoimmune diseases. Arch Intern Med 165:2337-2344 